\title{
Research on Code Division Multiple Access Based on Chirp Multi-Carrier
}

\author{
Zhenyu Jia ${ }^{1}$, Fei Yuan* ${ }^{1}$, Keyu Chen ${ }^{1}$, En Cheng ${ }^{1}$, Jianghui Li ${ }^{2}$ \\ ${ }^{\prime}$ Key Laboratory of Underwater Acoustic Communication and Marine Information Technology \\ Ministry of Education in Xiamen University \\ ${ }^{2}$ Institute of Sound and Vibration Research, University of Southampton \\ Southampton, United Kingdom \\ *Corresponding Author: yuanfei@xmu.edu.cn
}

\begin{abstract}
Underwater acoustic (UWA) channels are characterized by narrow-band, time-varying, and strong multipath interference. At the same time, Doppler effect also has a significant impact in UWA communications due to low sound speed in water. Therefore, traditional wireless multiple access technology cannot be used directly underwater. However, by combining some existing technologies in underwater communications, the traditional multiple access method can be improved to enable underwater multiple access. In this paper, we introduced chirp signals as carriers, which is widely used in UWA communications. Combined with code division multiple access (CDMA) and orthogonal frequency division multiple access (OFDMA) techniques, a multi-carrier chirp signal based CDMA method is proposed, which improves the reliability and spectrum efficiency of underwater multiple access. The simulation results show that the proposed method has good Bit error rate (BER) performance. Further, the method is also able to achieve good BER performance under the influence of Doppler effect because chirp signals is capable of resisting Doppler effects.
\end{abstract}

Keywords-Multiple Access, CDMA, Chirp, Sub-carriers.

\section{INTRODUCTION}

Code Division Multiple Access (CDMA), which possesses high system capacity and spectrum utilization, is a widely used multiple access method in second- and third-generation cellular systems [1]. It assigns mutually orthogonal address codes (pseudo-random codes) to different users, using their autocorrelation properties of the PN spreading code and the orthogonality between $\mathrm{PN}$ codes. At the receiving end, the information of different users is obtained by dispreading according to the orthogonal PN sequence [1].

Orthogonal Frequency Division Multiple Access (OFDMA) is a widely used multiple access method in $4 \mathrm{G}$ communications. It modulates different users respectively on different mutually orthogonal sub-carriers, that is, multiple users are allowed to send information simultaneously on different sub-carriers of each OFDM symbol. Mutually orthogonal carriers can significantly reduce interference between users. At the receiving end, different subcarriers are demodulated by discrete Fourier transform, and then the information of different users on each subcarrier is decoded [2].

Although CDMA system possesses higher system capacity, it is susceptible to mutual interference among users and has poor performance in the environment of frequency-selective fading. OFDMA technology, on the other hand, has better anti-user interference and frequency selective fading interference performance [3-5].

Linearity frequency modulation signal (chirp signal) is a kind of non-stationary signal, which is widely used in sonar detection, radar and communications [2]. Chirp signal occupies a large bandwidth and therefore has a good performance in selective fading channels. also, it has better anti-Doppler effect characteristics [8]. Currently, the communication systems based on chirp signal mainly adopt direct modulation (DM) and binary orthogonal keying (BOK) modulation [12].

In this paper, we study the multiple access based on multichirp carrier, and combined the traditional multi-carrier CDMA method with the chirp signal to improve the multiple access performance in UWA communication. First, the bit stream information to be sent is serial-to-parallel converted. Then, the data of different users are spread-modulated using PN codes that are orthogonal to each other. Finally, data of different users are modulated onto different OFDM subcarriers.

\section{SYSTEM MODEL}

Acoustic channels are characterized by severe multipath interference and fast phase changes. The multi-carrier chirp signal CDMA scheme [1] studied in this paper adopts OFDMA [2], ensuring high-speed data transmission and effectively avoiding overcoming various interferences in UWA channels. In the frequency domain subcarrier division, a higher spectrum utilization is allocated.

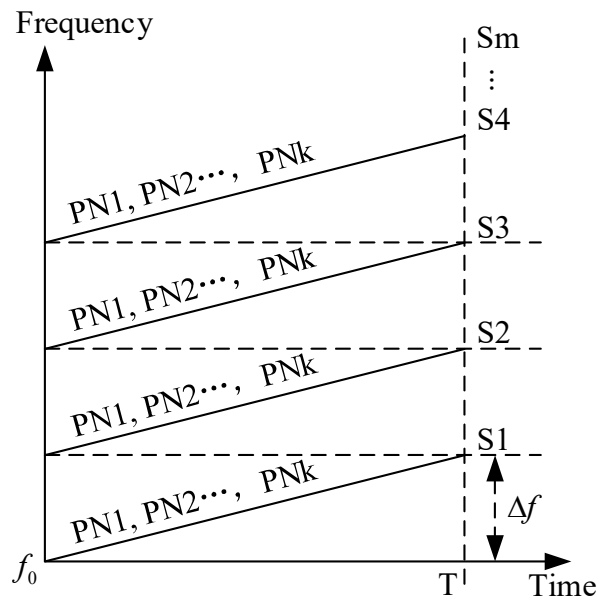

Fig. 1. Schematic of Multiple access 
As shown in Fig. 1, the orthogonal chirp signal is used as the carrier signal, which is easy to detect so that the system can reduce the misjudgment, therefore the system has high reliability. Secondly, using the orthogonal subcarrier, this system makes full use of the advantages of OFDM technology, therefore the system spectrum utilization is further improved. At the same time, users on different subcarriers use different PN sequences, reducing the mutual interference.

Fig. 2 shows that the bit stream data to be transmitted is firstly divided into several bit streams through serial-to-parallel conversion, data of different users are then spread by a different PN code, and then modulated onto different chirp subcarriers. From Fig. 2 and (1), we can see that these subcarriers have the same frequency modulation rate, but the center frequency is different, which overlaps each other in the frequency band to form an OFDM subcarrier. After the modulation is completed, the subcarriers are superimposed and then transmitted into the channel.

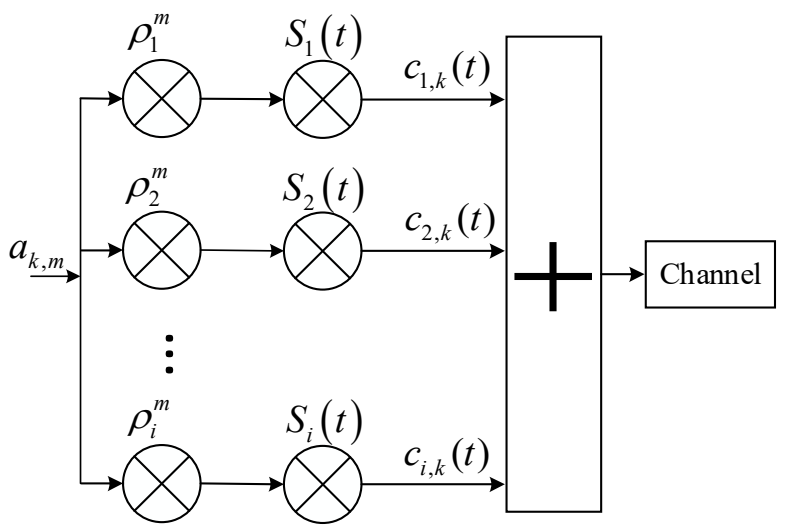

Fig. 2. Model of Sender.

Set the number of subcarriers in the system to N:

$$
S_{i}(t)=\cos \left(2 \pi\left(f_{0}+(i-1) \Delta f\right) t+\pi \mu t^{2}\right),
$$

where $S_{i}(t)$ represents the ith chirp subcarrier, $f_{0}$ is the first subcarrier $S_{1}(t)$ center frequency, $\Delta f$ represents the frequency interval between adjacent subcarriers, $f_{0}+i \Delta f$ is the center frequency of $S_{i}(t), \mu$ is the chirp rate, $T$ is the duration of the chirp subcarrier, $B$ is the bandwidth, and $\mu=$ $B / T$.

Let $a_{k}^{m} \in\{-1,1\}$ be the signal of the ith user at the moment, and all the signals of all the users at this moment are:

$$
\mathbf{A}=\left[\begin{array}{llll}
a_{k}^{0} & a_{k}^{1} & \ldots & a_{k}^{N-1}
\end{array}\right]^{T}
$$

Let $P^{m}$ be the mth user's spreading code:

$$
\boldsymbol{P}=\left[\begin{array}{cccc}
\rho_{0}^{0} & \rho_{0}^{1} & \ldots & \rho_{0}^{N-1} \\
\rho_{1}^{0} & \rho_{1}^{1} & \ldots & \rho_{1}^{N-1} \\
\vdots & \vdots & \ddots & \vdots \\
\rho_{N-1}^{0} & \rho_{N-1}^{1} & \ldots & \rho_{N-1}^{N-1}
\end{array}\right]=\left[\begin{array}{llll}
\boldsymbol{P}^{0} & \boldsymbol{P}^{1} & \ldots & \boldsymbol{P}^{N-1}
\end{array}\right]
$$

where $\rho_{i}^{m}$ is the spreading code corresponding to the user on the ith subcarrier.

After the modulation is completed, the sub-carriers are superimposed and then sent to the channel. The signal on the subcarrier is

$$
C_{(i, k)(t)}=\left[\sum_{m=0}^{N-1} a_{k}^{m} \rho_{i}^{m}\right] \cos \left(2 \pi\left(f_{0}+i \Delta f\right) t+\pi \mu t^{2}\right) g(t-k T)
$$

where $g(t)$ represents the unit pulse waveform.

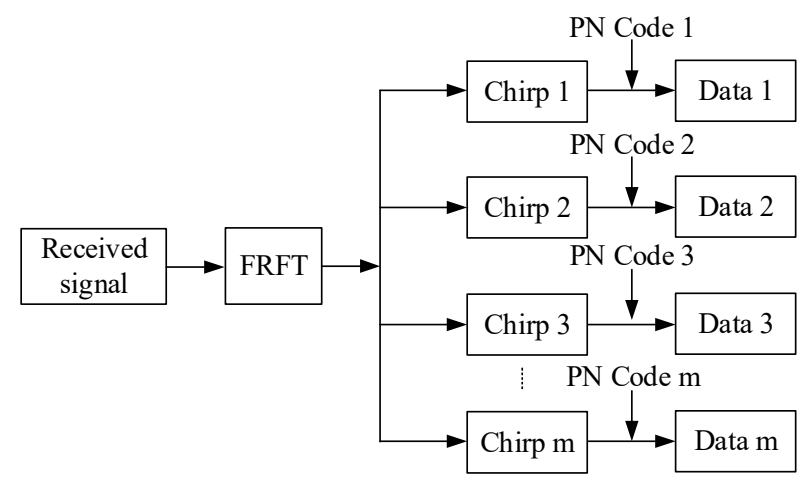

Fig. 3. Model of Receiver.

Fig. 3 shows that, after the signal is received, it firstly undergoes $\mathrm{A} / \mathrm{D}$ conversion to obtain the signal to be demodulated. The signal to be demodulated is detected and decided by the best fractional Fourier transform (FRFT), and then dispread by the PN code used by the modulation, so that the information sent by the user is obtained.

\section{THE ORTHOGONALITY OF CHIRP SUBCARRIERS}

According to the above, to distinguish different users by means of OFDMA subcarriers, we have to maintain the orthogonal relationship between these subcarrier. We can control the orthogonality by making the cross-correlation coefficient between subcarriers zero to reduce the multiple access interference between subcarriers.

The correlation coefficient between the ith chirp and the jth chirp subcarrier is as

$$
\rho_{l, m}=\frac{1}{T} \int_{-\frac{T}{2}}^{\frac{T}{2}} S_{i}^{*}(t) \cdot S_{m}(t) d t=\frac{1}{T} \frac{\sin [\pi(l-m) \Delta f T]}{\pi(l-m) \Delta f}
$$

When these two carriers meet the condition:

$$
(l-m) \Delta f=\frac{A}{T} \quad A \in Z,
$$

we can say that the cross-correlation coefficient between these two subcarriers is zero. 
Fig. 4 shows the cross-correlation coefficient between chirp signals, from which we can see that when the interval of center frequency of this set of chirp carriers meet the condition of

$$
\Delta f=1 / T=25 H z,
$$

the correlation coefficient between subcarriers is zero.

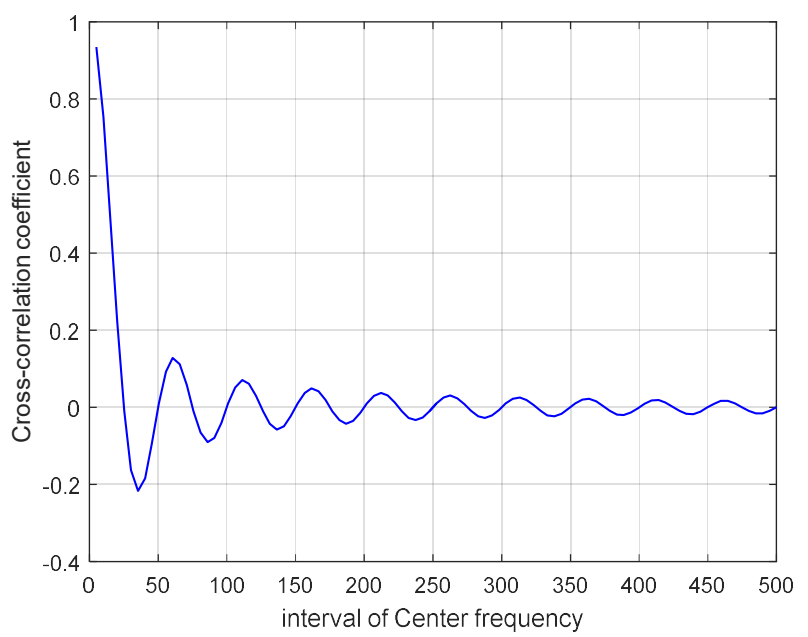

Fig. 4. Cross-correlation coefficient between Chirp signals

In the Gaussian channel, the bit-error-rate (BER) of chirpbok [8] system is given by

$$
P_{b}\left(\rho_{l, m}, \gamma_{b}\right)=Q(a, b)-\frac{1}{2} e^{-\frac{\left(a^{2}+b^{2}\right)}{2}} I_{o}(a b),
$$

where $Q(a, b)$ is the $\mathrm{Q}$ function, $I_{o}(a b)$ is the zero-order Bessel function, and $\gamma_{b}$ is the signal-to-noise ratio (SNR) per bit.

$$
\begin{aligned}
& a=\sqrt{\frac{\gamma_{b}}{2}\left(1-\sqrt{1-\left|\rho_{l, m}\right|^{2}}\right)} \\
& b=\sqrt{\frac{\gamma_{b}}{2}\left(1+\sqrt{1-\left|\rho_{l, m}\right|^{2}}\right)}
\end{aligned}
$$

Ideally, the chirp-bok system uses mutually orthogonal chirp signals, that is, $\rho=0$, and the BER can be given by

$$
P_{b}=\frac{1}{2} e^{-\frac{y_{b}}{2}}
$$

According to (8) and (11), given the cross-correlation coefficient of the chirp signal, the theoretical BER of the chirpbok system can be calculated based on the SNR of the received signal.

The simulation results are shown in Fig. 5. It can be seen that when the cross-correlation coefficient is in the range of $0 \sim 0.3$, the BER performance decreases slightly compared with the BER in the ideal condition, but the degree of attenuation is still within the acceptable range. However, when the correlation coefficient is greater than 0.3 , the performance degradation is very obvious, which shows that the correlation coefficient has a very significant impact on the performance of chirp-bok system.

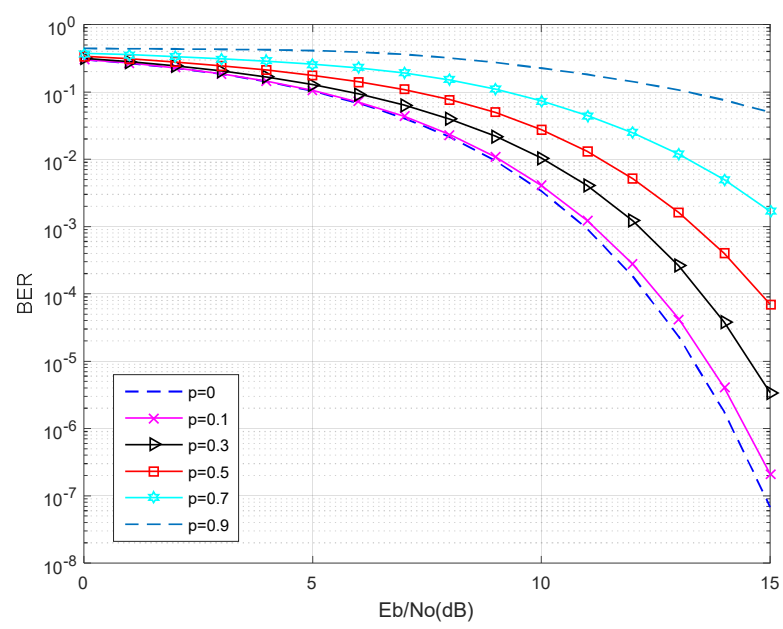

Fig. 5. BER performance of Chirp-bok system

\section{FRACTIONAL FOURIER TRANSFORM}

Currently, the main method used for demodulation of Chirp signals based communication system is FRFT (fractional Fourier transform) [3-5]. In the application of digital signal processing, discrete form of FRFT transform is required [6-7].

Fractional Fourier Transform (FRFT) is a generalization of traditional Fourier Transform. The traditional Fourier transform rotates the time axis $\pi / 2$ to the frequency axis. However, in the fractional domain, the rotation of the time axis any angle. Let time-domain signal is $x(t)$, then its p-order FRFT transform can be expressed as

$$
X_{p}(u)=F^{p}[x(t)] u(t)=\int_{-\infty}^{+\infty} x(t) K_{p}(t, u) d t
$$

and

$$
K_{p}(t, u)=\left\{\begin{array}{c}
A_{\alpha} \exp \left[j \pi\left(u^{2} \cot \alpha-2 u t \csc \alpha+t^{2} \cot \alpha\right)\right], \alpha \neq n \pi \\
\delta(u-t), \quad \alpha=2 n \pi \\
\delta(u+t), \quad \alpha=(2 n \pm 1) \pi
\end{array},\right.
$$

where $\alpha(\alpha=p \pi / 2)$ represents the rotation angle of the timefrequency axis, and $A_{\alpha}=\sqrt{1-j \cot \alpha}$.

Here, we perform a FRFT transform on a chirp signal with its center frequency $f_{0}=10 \mathrm{kHz}$, duration $T=10 \mathrm{~ms}$, and bandwidth $B=10 \mathrm{kHz}$. System sampling rate $f_{s}=40 \mathrm{kHz}$.

Fig. 6 shows the FRFT of the chirp signal. The waveform of chirp signal in the u-domain shows a clear energy focus [1]. Based on the impulse characteristic, we can estimate the parameters of the chirp signal [2]. 


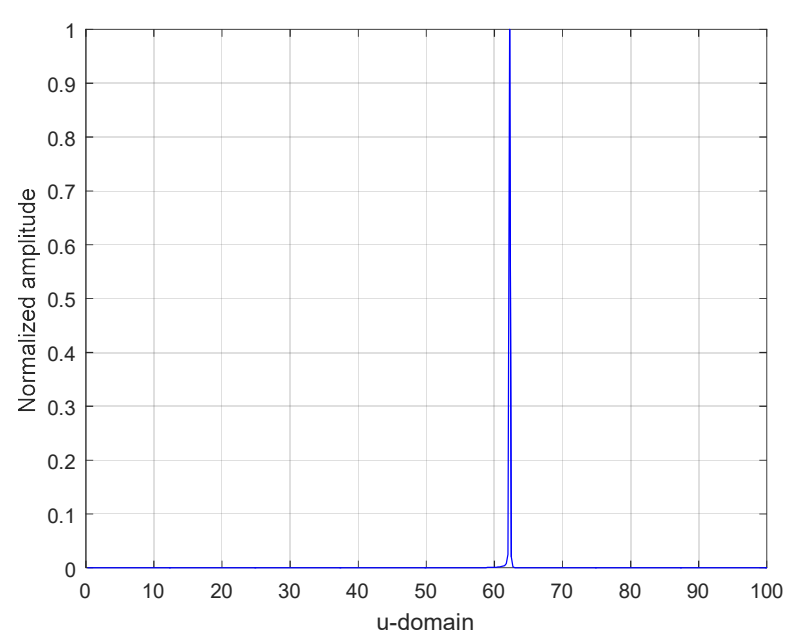

Fig. 6. FRFT of Chirp signal.

\section{SimUlATION}

\section{A. Different numbers of users}

Aiming at UWA channel, we choose the channel of $20 \mathrm{kHz} \sim 30 \mathrm{kHz}$ frequency band, the systematic sampling frequency is $90 \mathrm{kHz}$. Chirp signals of the same sweep range $B=4 \mathrm{kHz}$ are selected as subcarriers, and the center frequency range is $20 \mathrm{kHz} \sim 22 \mathrm{kHz}$.

TABLE I. SIMULATION PARAMETERS

\begin{tabular}{|c|c|c|c|}
\hline Number of users & 3 & 6 & 9 \\
\hline Interval ( $\mathrm{Hz})$ & 1000 & 500 & 250 \\
\hline Sweep bandwidth $(\mathrm{KHz})$ & 4 & 4 & 4 \\
\hline Duration of a chirp signal $(\mathrm{ms})$ & 20 & 20 & 20 \\
\hline Chirp rate $(\mathrm{KHz} / \mathrm{s})$ & 200 & 200 & 200 \\
\hline
\end{tabular}

TABLE I shows the parameters of different system. Each system has a different number of users and occupies the same bandwidth. In this way, neighboring chirp subcarriers on each system have different center frequency intervals. Also, a simulation is done using these system parameters. By setting a different number of users, the number of subcarriers divided in this frequency band and the center frequency between subcarriers are controlled. According to the above chirp subcarrier orthogonal conditions, the intervals of center frequency were selected $\Delta f=1000 \mathrm{~Hz}, \Delta f=500 \mathrm{~Hz}, \Delta f=$ $250 \mathrm{~Hz}$, so that we divided $3,5,9$ subcarriers within a limited bandwidth resources.

At the receiving end, the first thing is to perform the FRFT transform on the received signal. The optimal order of the FRFT transform is only related to the chirp rate $k$. Since the chirp signal shows a significant energy accumulation in the optimal FRFT domain, the location of this impulse in the u-domain is related to the center frequency of the chirp signal. Based on this, we only need to analyze the FRFT transformation of the received signal, we can separate the subcarriers of different users.
As shown in Fig. 7, when the number of chirp subcarriers is 3 , each subcarrier has the same chirp rate and different center frequency. After performing the optimal order FRFT transform, three peaks appear in the u-domain.

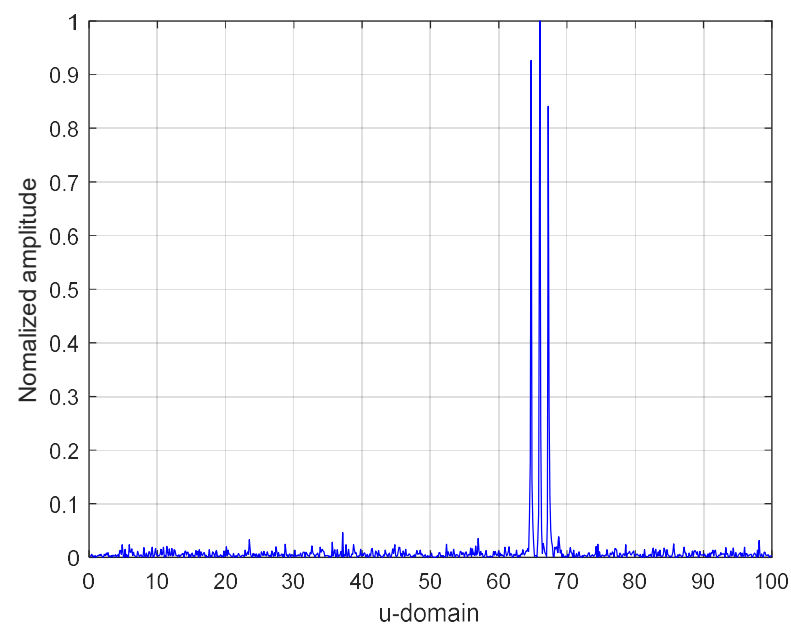

Fig. 7. FRFT of received signal

In this case, we can detect three chirp carriers with different center frequencies based on the positions of the three peaks in the u-domain. Then the data is demodulated and transmitted by the corresponding user on each subcarrier.

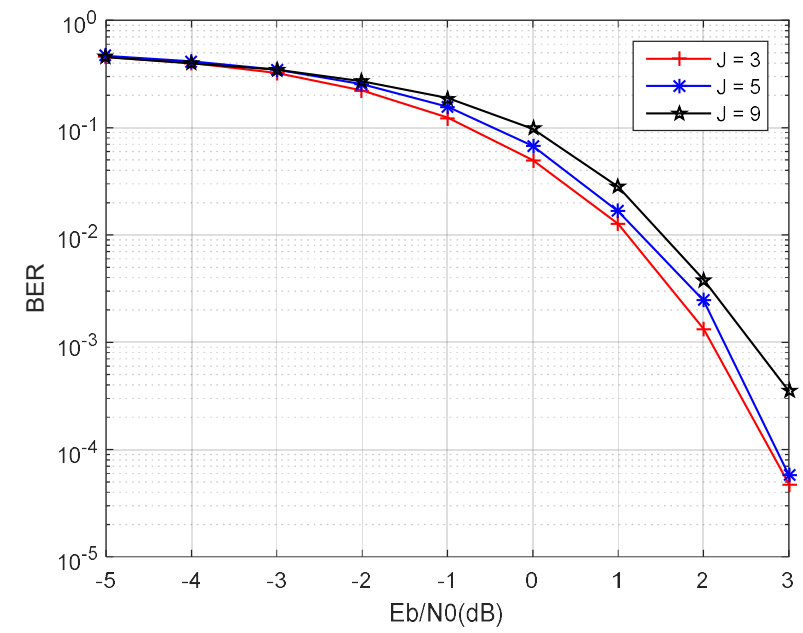

Fig. 8. BER performance

Fig. 8 shows the BER performance in AWGN (Additive White Gaussian Noise) channel. As the number of users increases, the number of sub-channels divided under the limited frequency band resources also increases, and the center frequency spacing between adjacent subcarriers decreases. Due to the limited sweep bandwidth of the chirp carriers, the smaller the center frequency interval is, the greater the overlap between the peak values of different subcarriers in the u-domain. This results in larger detection error and furthermore an increase in BER performance. 


\section{B. Doppler effect}

When the acoustic signal is transmitted, the difference of the frequency between the transmitted signal and the received signal due to the mutual movement between the sound source and the receiver is called Doppler effect (or the Doppler shift). In UWA communication, the signal is transmitted in water at a speed of about $1500 \mathrm{~m} / \mathrm{s}$, while in wireless communication the electromagnetic wave propagates through air at a speed of $3 \times 10^{8} \mathrm{~m} / \mathrm{s}$. Therefore, in contrast, the relative movement of the sound source and the receiver will be more obvious for the signal transmission, and the Doppler shift affects the quality of the communication more seriously.

Set the frequency of the transmitted signal from the sound source as $f_{0}$, affected by the Doppler effect, the frequency of the received signal as $f$, the relative velocity of the sound source and the receiver as $v$, the speed of the acoustic signal is $c$, the Doppler factor as $\beta$, the relationship between observed frequency $f$ and emitted frequency $f_{0}$ and the Doppler shift $f_{d}$ is given by

$$
\begin{gathered}
f_{d}=f-f_{0}=\frac{v}{c} f_{0}, \\
f=\left(1+\frac{v}{c}\right) f_{0}=\beta f_{0}
\end{gathered}
$$

Affected by the Doppler effect, the chirp signal received by the receiver is given by

$$
c(t)=\cos \left(2 \pi \beta f_{0}+\pi \beta^{2} \mu t^{2}\right),
$$

where $f_{0}$ is the center frequency of emitted chirp signal, $\mu$ is the chirp rate. As can be seen from (16), affected by the Doppler effect, the center frequency of the chirp signal received by the receiver becomes $\beta f_{0}$, and the chirp rate becomes $\beta^{2} \mu$. In order to show the impact of Doppler effect on chirp signal, we choose the chirp signal of duration $T=40 \mathrm{~ms}$, center frequency $f_{0}=$ $20 \mathrm{kHz}$ and sweep width $B=10 \mathrm{kHz}$. Set the relative moving speed of transmitter and receiver is $15 \mathrm{~m} / \mathrm{s}$. Simulation results shown in Fig. 9:
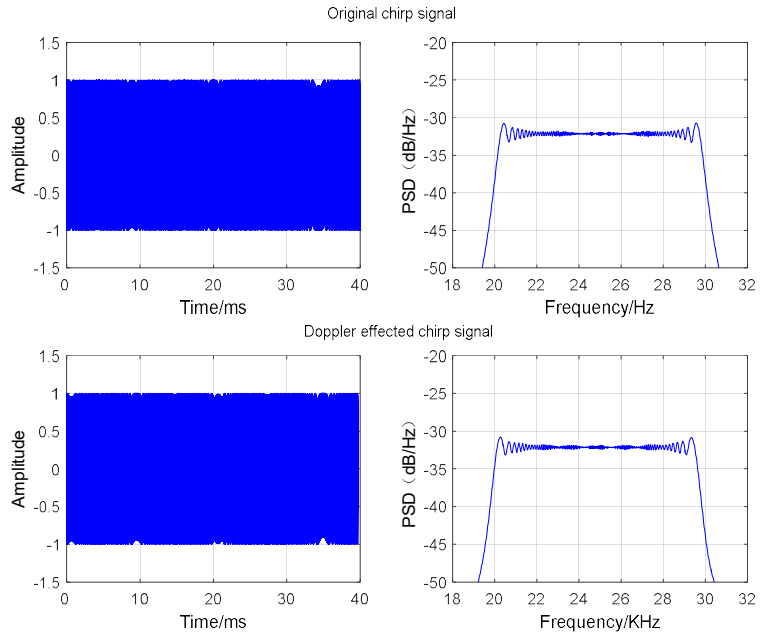

Fig. 9. Chirp signal under the influence of Doppler effect.
It can be seen from the figure that, affected by the Doppler effect, the chirp signal is compressed in time-domain, and has a certain degree of frequency offset.

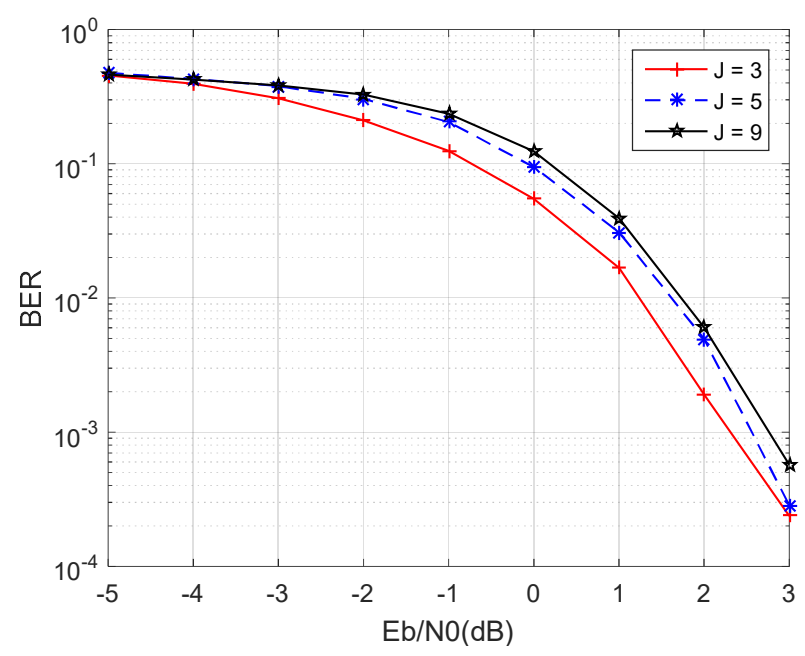

Fig. 10. BER performance in the simulation.

It can be seen from Fig.10, under the influence of the Doppler effect, all of the system performance has been reduced to some extent. This is because each chirp subcarrier is affected by the Doppler effect, and the received signals undergo different degrees of frequency offset at the receiving end. The larger the center frequency of the signal, the greater the amount of offset. The shift of the center frequency causes the waveform after receiving the FRFT transform to also have a certain degree of offset in the u-domain, thereby affecting the accuracy of the decision.

\section{ACKNOWLEDGMENT}

This work was supported by Key Laboratory of Universal Wireless Communications (Beijing University of Posts and Telecommunications), Ministry of Education, P. R. China (KFKT-2015101), the Natural Science Foundation of Fujian Province, China (Grant No. 2017J05109) and the National Natural Science Foundation of China (Grant No. 61571377, 61471308, 61771412).

\section{REFERENCES}

[1] A Kocian, D Dahlhaus. "Downlink performance analysis of a CDMA mobile radio system with chirp modulation". Vehicular Technology Conference:vol.1[C]. Houston: IEEE, 1999: 238-242.

[2] P. He, lv Yinghua, H. Zhang, et al. "SAW chirp Fourier transform for MB-OFDM UWB receiver[J]". "The journal of china universities of post and telecommunications", 2006, 13(3): 1-6.

[3] F. Liu, Y. Huang, R. Tao. "Chirp-rate resolution of fractional fourier transform in multi-component LFM signal[J]". Journal of Beijing Institute of Technology (English Edition), 2009, 18(1): 74-78.

[4] M. Ma, J. Cai, X. Zhang. "An estimation algorithm of chirp based on fractional fourier transform and frequency domain differential." International Conference on Computational Problem-Solving[C]. Lijiang: IEEE, 2010:218-221.

[5] R. Jacob, T. Thomas, A. Unnikrishnan. "Fractional fourier transform based chirp detector versus some conventional detectors." International Symposium on Ocean Electronics[C]. Cochin: IEEE, 2010: 56-65. 
[6] H M Ozaktas, M A Kutay, G. Bozdagi. "Digital computation of the fractional Fourier transform.” IEEE Trans. Sig. Proc., 1996; 44(9): 21412150.

[7] C Candan, M A Kutay, H M Ozaktas. "The discrete fractional Fourier transform.” IEEE Trans. Sig. Proc., 2000; 48(5): 1329-1337.

[8] X. Wang, M. Fei, X. Li, "Performance of Chirp Spread Spectrum in Wireless Communication Systems." IEEE Singapore International Conference on Communication[C], 2009, 55 (11) :466-469

[9] R. Tao, N. Zhang, Y. Wang. "Analysing and compensating the effects of range and Doppler frequency migrations in linear frequency modulation pulse compression radar[J]". Let Radar Sonar \& Navigation, 2011, $5(1): 12-22$.

[10] F. Meng, X. GU. "A Combined Chirp Signal Modulation Technique for Multiple Access System[J]". Information Technology Journal, 2011, 10(2): 416-421.
[11] P MOUNGNOUL, T H TRAN, T PAUNGMA. "Investigation of MultiLinear Chirp FH-CDMA over Fading Channel Model". The Fifth International Conference on Information Communications \& Signal Processing. Bangkok: IEEE Press, 2005:1480-1484.

[12] C. Lin, H. Wang, F. Yuan "An Improved Modulation Based on BOK in Underwater Acoustic Channel[J]". Advances in Information Sciences \& Service Sciences, 2013.

[13] H K Lakshminarayana, J S Bhat, B N Jagadale, et al. "Improved Chirp Modulation Spread Spectrum Receiver Based on Fractional Fourier Transform for Multiple Access[C]" International Conference on Signal Processing Systems. IEEE Computer Society, 2009:282-286.

[14] Q. Zhao, Q. Zhang, N. Zhang. "Multiple Chirp-Rate Modulation Based on Fractional Fourier Transform[C]" First International Conference on Pervasive Computing Signal Processing and Applications. IEEE, 2010:688-691. 